\title{
UN EJEMPLO DEL ESPECTRO DE FUCIK
}

\author{
Santiago César Rojas Romero \\ RESUMEN.- En el presente trabajo presentamos el Espectro de Fucik del \\ operador Laplaciano $-\Delta$ bajo condiciones de frontera tipo Dirichlet so- \\ bre un dominio $\Omega$ regular acotado en $R^{n}$, se demuestra el comporta- \\ miento asintótico de la primera curva contenida en él y se brinda una \\ descripción completa del Espectro de Fucik para el caso $n=1$.
}

\section{ESPECTRO DE FUCIK DEL LAPLACIANO PARA EL PROBLEMA TIPO DIRICHLET}

Sea $\Omega$ un dominio regular acotado en $R^{n}$. El espectro de Fucik del operador Laplaciano $-\Delta$ bajo condiciones de frontera tipo Dirichlet es definido como el conjunto $\sum$ de aquellos $(\alpha, \beta) \in R^{2}$; tales que el problema

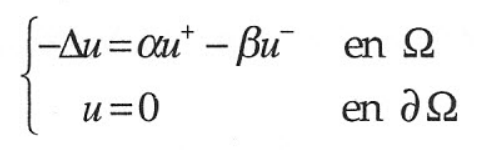

tiene una solución no trivial. Aquí $u^{+}=\max \{u, 0\}$ y $u^{-}=\min \{-u, 0\}$.

Es claro que haciendo $\alpha=\beta$ en (1) se tiene el espectro usual (dado que, $u=u^{+}-u^{-}$) y en ese caso $\alpha=\beta$ viene a ser uno de los autovalores de $-\Delta$, lo cual prueba que

$$
(\lambda, \lambda) \in \sum, \forall \lambda \text { autovalor de }-\Delta
$$

Luego, $\sum \neq \phi$.

En adelante llamaremos $\lambda_{1}$ al primer autovalor de $-\Delta$ sobre $H_{0}^{1}(\Omega)$ y $\varphi_{1}$ a su correspondiente autofunción positiva. No es difícil verificar la igualdad (2) que se usará mas adelante: 


$$
\int_{\Omega}\left|\nabla \varphi_{1}\right|^{2}=\lambda_{1} \int_{\Omega} \varphi_{1}^{2}
$$

Algunas propiedades de $\sum$

i) $\sum$ es simétrico respecto a la diagonal, es decir $(\alpha, \beta) \in \sum$ s.s.s. $(\beta, \alpha) \in \sum$. En efecto, debido a que $(-u)^{+}=u^{-}$y $(-u)^{-}=u^{+}$, si existe $u_{0}$ no trivial tal que

$$
-\Delta u_{0}=\alpha u_{0}^{+}-\beta u_{0}^{-} \quad \text { en } \Omega \quad \text { y } \quad u_{0}=0 \text { en } \partial \Omega
$$

entonces

$$
-\Delta \hat{u}_{0}=\beta \hat{u}_{0}^{+}-\alpha \hat{u}_{0}^{-} \quad \text { en } \Omega \quad \text { y } \quad \hat{u}_{0}=0 \text { en } \partial \Omega
$$

para $\hat{u}_{0}=-u_{0}$.

ii) Las rectas $\left\{\lambda_{1}\right\} \times \mathbb{R}$ y $\mathbb{R} \times\left\{\lambda_{1}\right\}$ estan contenidas en $\sum$. Por i), basta mostrar que $\left\{\lambda_{1}\right\} \times \mathbb{R} \subset \Sigma$. En efecto,

$$
-\Delta \varphi_{1}=\lambda_{1} \varphi_{1}^{+}-\beta \varphi_{1}^{-} \quad \text { en } \quad \Omega, \forall \beta \in \mathbb{R}
$$

pues $\varphi_{1}^{+}=\varphi_{1}$ y $\varphi_{1}^{-}=0$. Luego, $\left(\lambda_{1}, \beta\right) \in \sum \quad \forall \beta \in \mathbb{R}$.

\section{COMPORTAMIENTO ASINTÓTICO DE LA PRIMERA CURVA EN EL} ESPECTRO DE FUCIK DE $-\triangle$ PARA EL PROBLEMA TIPO DIRICHLET

Primero veamos la formulación variacional del problema (1). Si $u$ es solución de (1), entonces

$$
-\int_{\Omega} \Delta u \cdot v=\alpha \int_{\Omega} u^{+} v-\beta \int_{\Omega} u^{-} v, \forall v \in D(\Omega)
$$

luego

$$
\int_{\Omega} \nabla u \cdot \nabla v=\alpha \int_{\Omega} u^{+} v-\beta \int_{\Omega} u^{-} v, \forall v \in H_{0}^{1}(\Omega)
$$


De otro lado, se sabe que $-\Delta \varphi_{1} \cdot u=\lambda_{1} \varphi_{1}\left(u^{+}-u^{-}\right)$y de ahí que

$$
\int_{\Omega} \nabla \varphi_{1} \cdot \nabla u=\lambda_{1} \int_{\Omega} \varphi_{1} u^{+}-\lambda_{1} \int_{\Omega} \varphi_{1} u^{-},
$$

haciendo $v=\varphi_{1}$ en (3) se tiene

$$
\int_{\Omega} \nabla u \cdot \nabla \varphi_{1}=\alpha \int_{\Omega} u^{+} \varphi_{1}-\beta \int_{\Omega} u^{-} \varphi_{1}
$$

$\mathrm{y}$, restando las últimas igualdades

$$
\int_{\Omega} u^{+} \varphi_{1}-\frac{\beta-\lambda_{1}}{\alpha-\lambda_{1}} \int_{\Omega} u^{-} \varphi_{1}=0, \forall \alpha \neq \lambda_{1} .
$$

Ahora, para $r>0$, se consideran los conjuntos

$$
\begin{aligned}
& M_{r}=\left\{u \in H_{0}^{1}(\Omega): \int_{\Omega}\left(u^{+} \varphi_{1}-r u^{-} \varphi_{1}\right)=0\right\} \\
& N_{r}=\left\{u \in H_{0}^{1}(\Omega): \int_{\Omega}\left(u^{+}\right)^{2}+r\left(u^{-}\right)^{2}=1\right\} .
\end{aligned}
$$

y se definen

$$
\begin{aligned}
& \alpha=\alpha(r)=\lambda_{1}+\inf \left\{\int_{\Omega}\left(|\nabla u|^{2}-\lambda_{1} u^{2}\right): u \in M_{r} \cap N_{r}\right\} \\
& \beta=\beta(r)=\lambda_{1}+r \inf \left\{\int_{\Omega}\left(|\nabla u|^{2}-\lambda_{1} u^{2}\right): u \in M_{r} \cap N_{r}\right\} \\
& C_{1}=\left\{(\alpha(r), \beta(r)) \in \mathbb{R}^{2}: r>0\right\} .
\end{aligned}
$$

$C_{1}$ se denomina la primera curva en el espectro de Fucik.

Los siguientes resultados se demuestran adaptando los argumentos en [1]:

- $\alpha$ y $\beta$ definidos en (4) son mayores que $\lambda_{1}$. Además $(\alpha, \beta)$ es el primer punto de intersección de $\Sigma$ con la recta de pendiente $r$ y que pasa por $\left(\lambda_{1}, \lambda_{1}\right)$. 
- El ínfimo en (4) es alcanzado por las soluciones normalizadas (ie; soluciones en $N_{r}$ ) de (1).

- $C_{1}$ es una curva continua estrictamente decreciente y simétrica respecto a la diagonal.

- $\alpha(r) \rightarrow+\infty$ cuando $r \rightarrow 0$ y $\beta(r) \rightarrow+\infty$ cuando $r \rightarrow+\infty$.

Respecto al comportamiento asintótico de la curva $C_{1}$ se tiene el siguiente resultado

\section{Proposición 1.}

$\alpha(r) \rightarrow \lambda_{1}$ cuando $r \rightarrow+\infty$ y $\beta(r) \rightarrow \lambda_{1}$ cuando $r \rightarrow 0$

Demostración. De la definición (4) se verifica directamente

$$
\inf \left\{\int_{\Omega}\left(|\nabla u|^{2}-\lambda_{1} u^{2}\right): u \in M_{1 / r} \cap N_{1 / r}\right\}=r \inf \left\{\int_{\Omega}\left(|\nabla u|^{2}-\lambda_{1} u^{2}\right): u \in M_{r} \cap N_{r}\right\}
$$

y de ahi que $\alpha(r)=\beta(1 / r)$. Por ello, basta demostrar que $\alpha(r) \rightarrow \lambda_{1}$ cuando $r \rightarrow+\infty$, ie; $\forall \eta>0 \exists M>0$ tal que $\alpha(r)-\lambda_{1}<\eta, \forall r \geq M$. Supongamos por contradicción que $\exists \eta>0$ tal que $\alpha(r)-\lambda_{1} \geq \eta, \forall r>0$, entonces

$$
\inf \left\{\int_{\Omega}\left(|\nabla u|^{2}-\lambda_{1} u^{2}\right): u \in M_{r} \cap N_{r}\right\} \geq \eta
$$

lo cual implica que

$$
\int_{\Omega}\left(|\nabla u|^{2}-\lambda_{1} u^{2}\right) \geq \eta
$$

para todo $u \in H_{0}^{1}(\Omega)$ tal que, para algún $r>0, u \in M_{r} \cap N_{r}$.

Ahora, para $\varepsilon>0$ suficientemente pequeño, descomponemos $\Omega$ en $\hat{\Omega}=\hat{\Omega}_{\varepsilon}=\left\{x \in \Omega ; \operatorname{dist}\left(x, \Omega^{c}\right)>\varepsilon\right\}, \quad \tilde{\Omega}=\tilde{\Omega}_{\varepsilon}=\left\{x \in \Omega ; \operatorname{dist}\left(x, \Omega^{c}\right)<\varepsilon\right\}$ y denotemos con $\hat{\lambda}_{1}=\hat{\lambda}_{1, \varepsilon}, \tilde{\lambda}_{1}=\tilde{\lambda}_{1, \varepsilon}$ a los principales autovalores de $-\Delta$ sobre $H_{0}^{1}(\hat{\Omega}), H_{0}^{1}(\tilde{\Omega})$ respectivamente, y por $\hat{\varphi}_{1}=\hat{\varphi}_{1, \varepsilon}, \tilde{\varphi}_{1}=\tilde{\varphi}_{1, \varepsilon}$ a las correspondientes autofunciones positivas normalizadas en $L^{2}$, que se extienden con cero fuera de $\hat{\Omega}, \mathrm{y} \tilde{\Omega}$, respectivamente. Para $\delta>0$, definimos 


$$
u=u_{\varepsilon, \delta}=\left\{\begin{array}{rr}
\hat{\varphi}_{1} & \text { en } \hat{\Omega}, \\
-\delta \tilde{\varphi}_{1} & \text { en } \hat{\Omega} .
\end{array}\right.
$$

Entonces $u=\hat{\varphi}_{1}-\delta \tilde{\varphi}_{1} \in H_{0}^{1}(\Omega)$ y se tiene

$$
\begin{aligned}
\int_{\Omega}\left(|\nabla u|^{2}-\lambda_{1} u^{2}\right) & =\int_{\hat{\Omega}}\left(\left|\nabla \hat{\varphi}_{1}\right|^{2}-\lambda_{1} \hat{\varphi}_{1}^{2}\right)+\delta^{2} \int_{\tilde{\Omega}}\left(\left|\nabla \tilde{\varphi}_{1}\right|^{2}-\lambda_{1} \tilde{\varphi}_{1}^{2}\right)= \\
& =\left(\hat{\lambda}_{1}-\lambda_{1}\right)+\delta^{2}\left(\tilde{\lambda}_{1}-\lambda_{1}\right) .
\end{aligned}
$$

Como $\hat{\lambda}_{1}=\hat{\lambda}_{1, \varepsilon} \rightarrow \lambda_{1}$ cuando $\varepsilon \rightarrow 0$ (vea [1], Lema 2.5), elegimos $\varepsilon>0$, tal que $\hat{\lambda}_{1}-\lambda_{1}<\eta / 4$ y $\delta>0$ tal que $\tilde{\lambda}_{1}-\lambda_{1}<\eta / 4 \delta^{2}$. Con estos valores de $\varepsilon$ y $\delta$, se tiene

$$
\int_{\Omega}\left(|\nabla u|^{2}-\lambda_{1} u^{2}\right)<\eta / 2 .
$$

Por otro lado, se observa que $u \in M_{r}$ para algún $r=r(\varepsilon, \delta)$ apropiado pues $u$ cambia de signo. Además

$$
\begin{aligned}
\int_{\Omega}\left(\left(u^{+}\right)^{2}+r\left(u^{-}\right)^{2}\right) & =\int_{\hat{\Omega}} \hat{\varphi}_{1}^{2}+r \delta^{2} \int_{\widetilde{\Omega}} \tilde{\varphi}_{1}^{2} \\
& =1+r \delta^{2}>1 .
\end{aligned}
$$

Entonces $u / \sqrt{1+r \delta^{2}} \in N_{r}$. Por tanto, se ha construido una función que satisface las dos condiciones en el ínfimo (5) y verifica (7), lo cual es una contradicción con (6).

\section{EJEMPLO DEL ESPECTRO DE FUCIK}

En esta sección se dará una descripción completa del espectro de Fucik de para $\Omega=\langle 0, \pi\rangle \subset \mathbb{R}$; es decir, hallaremos el conjunto de puntos $(\alpha, \beta) \in \mathbb{R}^{2}$ para los cuales el problema

$$
\left\{\begin{array}{l}
-u^{\prime \prime}=\alpha u^{+}-\beta u^{-} \text {en }\langle 0, \pi\rangle \\
\mathrm{u}(0)=u(\pi)=0
\end{array}\right.
$$

tiene solución no trivial. 
En primer lugar, calcularemos $\lambda_{1}$ y $\varphi_{1}$ obtener primer autovalor y su correspondiente autofunción positiva de $-\Delta$ respectivamente. Para hallar $\lambda_{1}$ resolvemos la ecuación

$$
-u^{\prime \prime}=\lambda u \text { en }\langle 0, \pi\rangle
$$

Las soluciones de (9) tienen la forma

$$
\begin{cases}u(x)=A \operatorname{sen} \sqrt{\lambda} x+B \cos \sqrt{\lambda} x & \text { para } \lambda>0 \\ u(x)=A x+B & \text { para } \lambda=0 \\ u(x)=A e^{\sqrt{-\lambda} x}+B e^{-\sqrt{-\lambda} x} & \text { para } \lambda<0\end{cases}
$$

De ahí que una solución no trivial de (9) con las condiciones de frontera del problema (8) debe tener la forma

$$
u(x)=A \operatorname{sen} \sqrt{\lambda} x, \quad \lambda=n^{2}, n \in \mathbb{N}, \quad A \neq 0
$$

Así, el primer autovalor de $-D^{2}$ es $\lambda_{1}=1$ y la correspondiente autofunción positiva normalizada es $\varphi_{1}=\sqrt{\frac{2}{\pi}} \operatorname{sen} x$.

Lema. Sean $\alpha, \beta \in \mathbb{R}$, entonces el problema (8) tiene una solución no trivial si y sólo si, se verifica una de las siguientes condiciones:

i) $\alpha=1, \beta \in \mathbb{R}$ arbitrario;

ii) $\beta=1, \alpha \in \mathbb{R}$ arbitrario;

iii) $\alpha>1, \beta>1, \frac{\sqrt{\alpha} \sqrt{\beta}}{\sqrt{\alpha}+\sqrt{\beta}} \in \mathbb{N}$;

iv) $\alpha>1, \beta>1, \frac{\sqrt{\beta}(\sqrt{\alpha}-1)}{\sqrt{\alpha}+\sqrt{\beta}} \in \mathbb{N}$;

v) $\alpha>1, \beta>1, \frac{\sqrt{\alpha}(\sqrt{\beta}-1)}{\sqrt{\alpha}+\sqrt{\beta}} \in \mathbb{N}$.

Demostración. Sea $u_{0}$ una solución no trivial del problema (8).

Si $u_{0}$ no tiene ceros en el intervalo $\langle 0, \pi\rangle$, entonces $u_{0}$ es solución de la ecuación

$$
-u^{\prime \prime}-\lambda u=0
$$


donde $\lambda=\alpha$ si $u_{0}(x)>0, \forall x \in\langle 0, \pi\rangle$ o $\lambda=\beta$ si $u_{0}(x)<0, \forall x \in\langle 0, \pi\rangle$.

Entonces, de acuerdo con (11), $\lambda=n^{2}$ y $u_{0}(x)=A \operatorname{sen} n x$, pero como $u_{0}$ no tiene ceros en $\langle 0, \pi\rangle$, debe ser $\lambda=1$ y $u_{0}(x)=A \operatorname{sen} x$. En consecuencia, se verifican i) o ii).

Ahora veamos el caso en que $u_{0}$ tiene ceros en el intervalo $[0, \pi]$. Primero vemos que el número máximo de ceros es a lo más un número finito. Sea $x_{0}$ uno de los ceros de $u_{0}$, entonces $u_{0}^{\prime}\left(x_{0}\right) \neq 0$ (de lo contrario tendríamos que $u_{0}=0$ en $\langle 0, \pi\rangle$, pues para cada $\lambda>0$, la única solución del PVI

$$
\left\{\begin{array}{l}
-u^{\prime \prime}-\lambda u=0, \quad \text { en }\langle 0, \pi\rangle \\
u\left(x_{0}\right)=u^{\prime}\left(x_{0}\right)=0
\end{array}\right.
$$

es trivial). Luego, existe una vecindad del punto $x_{0}$ que no contiene otro cero de $u_{0}$. De lo anterior, y por la compacidad de $[0, \pi]$, se concluye que $u_{0}(x)$ debe tener sólo un número finito de ceros en $[0, \pi]$. Sea $u_{1}(x)$ la parte positiva de $u_{0}(x)$, entonces $u_{1}(x)$ es solución de

$$
-u^{\prime \prime}-\alpha u=0
$$

y por ello, $u_{1}(x)=A \operatorname{sen} \sqrt{\alpha}(x-\xi)$, con $A>0, \alpha>1$, $\xi$ cero de $u_{0}$.

Si $u_{2}(x)$ es la parte negativa de $u_{0}(x)$, entonces $u_{2}(x)$ es solución de

$$
-u^{\prime \prime}-\beta u=0
$$

y de ahí $u_{2}(x)=B \operatorname{sen} \sqrt{\beta}(x-\omega)$, con $B<0, \beta>1, \omega$ cero $u_{0}$.

Mas exactamente; si $\alpha \geq \beta$

$$
u_{0}(x)= \begin{cases}A \operatorname{sen} \sqrt{\alpha} x & , x \in\langle 0,\rangle \\ -\frac{\sqrt{\alpha}}{\sqrt{\beta}} A \operatorname{sen} \sqrt{\beta}\left(x-\frac{\pi}{\sqrt{\alpha}}\right) & , x \in\left\langle\frac{\pi}{\sqrt{\alpha}}, \frac{\pi}{\sqrt{\alpha}}+\frac{\pi}{\sqrt{\beta}}\right] \\ A \operatorname{sen} \sqrt{\alpha}\left(x-\frac{\pi}{\sqrt{\alpha}}-\frac{\pi}{\sqrt{\beta}}\right) & , x \in\left\langle\frac{\pi}{\sqrt{\alpha}}+\frac{\pi}{\sqrt{\beta}}, \frac{2 \pi}{\sqrt{\alpha}}+\frac{\pi}{\sqrt{\beta}}\right] \\ -\frac{\sqrt{\alpha}}{\sqrt{\beta}} A \operatorname{sen} \sqrt{\beta}\left(x-\frac{2 \pi}{\sqrt{\alpha}}-\frac{\pi}{\sqrt{\beta}}\right) & , x \in\left\langle\frac{2 \pi}{\sqrt{\alpha}}+\frac{\pi}{\sqrt{\beta}}, \frac{2 \pi}{\sqrt{\alpha}}+\frac{2 \pi}{\sqrt{\beta}}\right] \\ \vdots & \end{cases}
$$


con $A>0$. Y si $\alpha<\beta$

$$
u_{0}(x)= \begin{cases}B \operatorname{sen} \sqrt{\beta} x & , x \in\left\langle 0, \frac{\pi}{\sqrt{\beta}}\right\rangle \\ -\frac{\sqrt{\beta}}{\sqrt{\alpha}} B \operatorname{sen} \sqrt{\alpha}\left(x-\frac{\pi}{\sqrt{\beta}}\right) & , x \in\left\langle\frac{\pi}{\sqrt{\beta}}, \frac{\pi}{\sqrt{\alpha}}+\frac{\pi}{\sqrt{\beta}}\right] \\ B \operatorname{sen} \sqrt{\beta}\left(x-\frac{\pi}{\sqrt{\alpha}}-\frac{\pi}{\sqrt{\beta}}\right) & , x \in\left\langle\frac{\pi}{\sqrt{\alpha}}+\frac{\pi}{\sqrt{\beta}}, \frac{\pi}{\sqrt{\alpha}}+\frac{2 \pi}{\sqrt{\beta}}\right] \\ -\frac{\sqrt{\beta}}{\sqrt{\alpha}} B \operatorname{sen} \sqrt{\alpha}\left(x-\frac{\pi}{\sqrt{\alpha}}-\frac{2 \pi}{\sqrt{\beta}}\right) & , x \in\left\langle\frac{\pi}{\sqrt{\alpha}}+\frac{2 \pi}{\sqrt{\beta}}, \frac{2 \pi}{\sqrt{\alpha}}+\frac{2 \pi}{\sqrt{\beta}}\right] \\ \vdots & \end{cases}
$$

con $B<0$. En ambos casos, debe existir $k \in \mathbb{N}$ tal que se cumple una de las siguientes igualdades

$$
\begin{gathered}
k\left(\frac{\pi}{\sqrt{\alpha}}+\frac{\pi}{\sqrt{\beta}}\right)=\pi \quad, \quad k\left(\frac{\pi}{\sqrt{\alpha}}+\frac{\pi}{\sqrt{\beta}}\right)+\frac{\pi}{\sqrt{\alpha}}=\pi, \\
k\left(\frac{\pi}{\sqrt{\alpha}}+\frac{\pi}{\sqrt{\beta}}\right)+\frac{\pi}{\sqrt{\beta}}=\pi,
\end{gathered}
$$

lo cual significa que debe verificarse al menos una de las condiciones iii), iv) o v).

Recíprocamente, si se verifica i) o ii), $u(x)=A \operatorname{sen} x$ es solución del problema (8), con $A>0$ o $A<0$. Si se verifica una de las condiciones iii), iv) o v), entonces se cumple (14) con $k \in \mathbb{N}$; luego $u(x)$ en una de las formas, (12) o (13) es solución del problema (8).

Proposición 2. El espectro de Fucik de $-\Delta^{2}$ con condiciones de frontera tipo Dirichlet en el intervalo $\langle 0, \pi\rangle$ esta formado por todos aquellos $(\alpha, \beta) \in \mathbb{R}^{2}$ que verifican una de las condiciones del lema anterior.

Proposición 3. El espectro de Fucik de $-\Delta^{2}$ con condiciones de frontera tipo Dirichlet en el intervalo $\langle 0, c\rangle$ está formado por todos aquellos $(\alpha, \beta) \in \mathbb{R}^{2}$ que satisfacen una de las siguientes condiciones:

i) $\alpha=\frac{\pi^{2}}{c^{2}}, \beta \in \mathbb{R}$ arbitrario;

ii) $\alpha \in \mathbb{R}$ arbitrario, $\beta=\frac{\pi^{2}}{c^{2}}$;

iii) $\alpha>\frac{\pi^{2}}{c^{2}}, \beta>\frac{\pi^{2}}{c^{2}}, \exists k \in \mathbb{N} / k\left(\frac{\pi}{\sqrt{\alpha}}+\frac{\pi}{\sqrt{\beta}}\right)=c$; 
iv) $\alpha>\frac{\pi^{2}}{c^{2}}, \beta>\frac{\pi^{2}}{c^{2}}, \exists k \in \mathbb{N} / k\left(\frac{\pi}{\sqrt{\alpha}}+\frac{\pi}{\sqrt{\beta}}\right)+\frac{\pi}{\sqrt{\alpha}}=c$;

v) $\alpha>\frac{\pi^{2}}{c^{2}}, \beta>\frac{\pi^{2}}{c^{2}}, \exists k \in \mathbb{N} / k\left(\frac{\pi}{\sqrt{\alpha}}+\frac{\pi}{\sqrt{\beta}}\right)+\frac{\pi}{\sqrt{\beta}}=c$.

\section{BIBLIOGRAFÍA}

[1] De Figuereido, D.; J. P. Gossez. On the first curve of the Fucik Spectrum of an elliptic operator. Diff. int. eq., volume 7, 1285-1302 (1994).

[2] Espinoza, P. C. Discrete Analogue of Fucik Spectrum of the Laplacian. Journal of Computational and Applied Mathematics 103, 93 - 97 (1999).

[3] Fucik, S.; Kufner, A. Nonlinear Differential Equations. Elsevier Scientific Publishing Company. The Netherlands, (1980). 